\title{
BMJ Open Suitability of the German version of the Manchester Triage System to redirect emergency department patients to general practitioner care: a prospective cohort study
}

Anna Slagman, ${ }^{1,2}$ Felix Greiner, ${ }^{3}$ Julia Searle, ${ }^{2}$ Linton Harriss, ${ }^{1}$ Fintan Thompson, ${ }^{1}$ Johann Frick, ${ }^{2,4}$ Myrto Bolanaki, ${ }^{2}$ Tobias Lindner, ${ }^{2}$ Martin Möckel ${ }^{\oplus 1,2}$

To cite: Slagman A, Greiner F, Searle J, et al. Suitability of the German version of the Manchester Triage System to redirect emergency department patients to general practitioner care: a prospective cohort study. BMJ Open 2019;9:e024896. doi:10.1136/ bmjopen-2018-024896

- Prepublication history and additional material for this paper are available online. To view these files, please visit the journal online (http://dx.doi. org/10.1136/bmjopen-2018024896).

Received 20 June 2018 Revised 14 February 2019 Accepted 1 March 2019

Check for updates

(C) Author(s) (or their employer(s)) 2019. Re-use permitted under CC BY-NC. No commercial re-use. See rights and permissions. Published by BMJ.

For numbered affiliations see end of article.

Correspondence to

Dr Anna Slagman;

anna.slagman@jcu.edu.au

\section{ABSTRACT}

Objectives To investigate the suitability of the German version of the Manchester Triage System (MTS) as a potential tool to redirect emergency department (ED) patients to general practitioner care. Such tools are currently being discussed in the context of reorganisation of emergency care in Germany.

Design Prospective cohort study.

Setting Single centre University Hospital Emergency Department

Participants Adult, non-surgical ED patients.

Exposure A non-urgent triage category was defined as a green or blue triage category according to the German version of the MTS.

Primary and secondary outcome measures Surrogate parameters for short-term risk (admission rate, diagnoses, length of hospital stay, admission to the intensive care unit, in-hospital and 30-day mortality) and long-term risk (1-year mortality).

Results A total of 1122 people presenting to the ED participated in the study. Of these, $31.9 \%(n=358)$ received a non-urgent triage category and $68.1 \%(n=764)$ were urgent. Compared with non-urgent ED presentations, those with an urgent triage category were older (median age 60 vs 56 years, $p=0.001$ ), were more likely to require hospital admission (47.8\% vs $29.6 \%$ ) and had higher inhospital mortality (1.6\% vs $0.8 \%)$. There was no significant difference observed between non-urgent and urgent triage categories for 30 -day mortality $(1.2 \%[n=4]$ vs $2.2 \%$ $[n=15] ; p=0.285)$ or for 1 -year mortality $(7.9 \%[n=26]$ vs $10.5 \%[n=72] ; p=0.190)$. Urgency was not a significant predictor of 1-year mortality in univariate $(\mathrm{HR}=1.35$; $95 \% \mathrm{Cl} 0.87$ to $2.12 ; \mathrm{p}=0.185)$ and multivariate regression analyses $(\mathrm{HR}=1.20 ; 95 \% \mathrm{Cl} 0.77$ to $1.89 ; \mathrm{p}=0.420)$.

Conclusions The results of this study suggest the German MTS is unsuitable to safely identify patients for redirection to non-ED based GP care.

Trial registration number U1111-1119-7564; Postresults

\section{INTRODUCTION}

Emergency departments (EDs) worldwide and in Germany are facing increased patient

\section{Strengths and limitations of this study}

- This is the first study of its kind to examine in-hospital course, diagnoses and long-term mortality in adult, non-surgical emergency department (ED) patients with low urgency according to the German Manchester Triage System. Short-term and longterm outcomes were investigated.

- Clinical information from the ED and initial hospital stay were linked with long-term mortality data to provide additional information on long-term risk. The linkage of routine data from the hospital information system and the local register of residents constitutes a methodically innovative approach.

- Short-term mortality was low and thus case numbers may be insufficient to identify possible differences in short-term mortality.

- Sensitivity analyses indicate that more severe cases may have been excluded from the study, thus possibly effecting risk assessment estimation in the higher risk categories (urgent cases). However, this did not impact the risk assessment in non-urgent cases.

numbers and healthcare structures need to be adapted to prevent overcrowding and ensure high-quality patient care. ${ }^{1}$ Due to the increasing number of non-urgent ambulatory patients (ie, patients not in need of hospital admission for inpatient treatment) seeking emergency services, emergency care has emerged as one of the main issues of healthcare politics in Germany.

Interventions aimed at reducing the number of ED presentations have been discussed in the scientific literature. Gatekeeping may be one potential intervention to reduce ED crowding with a focus on reducing the number of non-urgent cases. These have been assumed to constitute a subgroup of 'general practitioner-type (GP-type)' or even 'inappropriate' ED visits. ${ }^{12}$ The current 
German government has committed to improve emergency care by implementing integrated emergency and coordination centres, run by a cooperation of inpatient and outpatient services with shared financing responsibilities. ${ }^{3}$ In these centres, gatekeeping tools are being implemented to identify patients who could be safely referred to GP care. In addition to determining acuity, these tools should determine sector of care and the appropriate medical specialty for presenting patients. ${ }^{4}$ Following this process, patients are assigned either to the emergency department or to outpatient (general practitioner [GP]) services. These GP care services may be in close proximity to the hospital, but however, may not have access to hospital-based resources. Similarly, patients may be referred to an out-of-hospital setting in another location. The general concern with this approach is that the Manchester Triage System (MTS) has never been evaluated for this purpose and valid and effective instruments to identify such 'GP-type patients' are still lacking. ${ }^{2}$ However, suggestions have been made to utilise the initial ED triage category to redirect patients to alternative care structures. ${ }^{5}$ To date, little evidence is available on the details of hospital stay (eg, length of stay [LOS], intensive care unit [ICU] admission, final discharge diagnosis) and other outcomes of patients with non-urgent ED triage category. This applies in particular on the suitability of the initial ED triage category as a gatekeeping tool for patient redirection to other healthcare structures, which is not the primary purpose of the ED triage system.

We investigated the suitability of the German version of the MTS as a gatekeeping tool for adult, non-surgical patients requiring emergency care by predicting shortterm and long-term outcomes such as admission to hospital or ICU, length of hospital stay, severity of diagnosis and mortality (in-hospital, 30-day and 1-year).

\section{METHODS \\ Study design}

Adult, non-surgical patients who presented to the ED of Charité Universitätsmedizin Berlin, Campus Virchow Klinikum between the 22 December 2010 and the 28 November 2011 were enrolled. Patients were eligible for study participation if they were aged $\geq 18$ years, the reason for presentation was non-surgical in nature, they were able to provide written informed consent and were not dependents of the principal investigator. Patients were recruited around the clock, 7 days a week and approached by research staff in the ED after first contact with the treating physician. Patients were only enrolled once during the study period, so subsequent presentations to the ED were excluded from analyses. This was a single-centre study based on linked routine secondary administrative data from the ED information system (EDIS), the hospital information system (HIS) and the 'Berlin register of residents'.

\section{Study setting}

The Charité University Hospital at Campus Virchow Klinikum provided tertiary care to $>80000$ patients during the study period. The hospital is one of three university campuses of Charité Universitätsmedizin Berlin, with 3000 hospital beds in total. At Campus Virchow Klinikum, the emergency care facilities are divided in three distinct sections: non-surgical emergency care (20461 cases during the study period), surgical emergency care (35888 cases) and paediatric emergency care (28614 cases). Patients selected for this study were recruited from the non-surgical emergency care track only.

\section{Outcome measures}

Surrogate parameters for short-term risk were admission to hospital after ED care, diagnoses (International Classfication of Disease [ICD]-10), length of hospital stay, admission to ICU, in-hospital mortality and 30-day mortality. Long-term risk was assessed by 1-year mortality.

\section{Data selection}

After enrolment, data on patient characteristics, clinical course in the ED, ED triage category and final primary ED diagnoses were retrieved from the EDIS for all patients. For admitted patients, additional information on final in-hospital diagnoses at hospital discharge and in-hospital course were retrieved from the HIS and linked to the EDIS data. Thirty-day and 1-year mortality was assessed in the Berlin register of residents using name, gender and date of birth as identifiers. Mortality was recorded as unknown if patients were not registered as residents in Berlin or if the information was not accessible.

\section{Definition of non-urgent ED visits}

A non-urgent ED visit was defined based on the initial triage category of the patient. At the participating ED, the German MTS is used to determine the maximum waiting time until first physician contact. ${ }^{6}$ Triage is conducted on presentation to the ED by a trained nurse. The German MTS divides patients into five categories according to a maximum waiting time recommended before first physician contact: red $=$ 'immediate resuscitation' $(0 \mathrm{~min})$, orange $=$ 'very urgent' $(10 \mathrm{~min})$, yellow $=$ 'urgent' $(30 \mathrm{~min})$, green $=$ 'standard' $(90 \mathrm{~min})$, blue $=$ 'non-urgent' (120 min). The German MTS times differ from the original British version, which has longer wait times for the yellow to blue categories. A non-urgent triage category in the current study was defined as a green or blue triage category, the other categories (red, orange, yellow) were classified as urgent. Triage was not conducted for those patients who are directly seen by an ED physician and triage category is missing for these patients in the current study. Triage determines the time between admission and first contact to an ED physician and thus is not necessary for patients who are directly referred to an ED physician. This applies predominately to patients who are transported to the ED by ambulance and are directly presented to an ED physician by the ambulance staff. 


\section{Statistical analysis}

As described above, a non-urgent triage category in the underlying analysis was defined as a green or blue triage category, the other categories (red, orange, yellow) were regarded as urgent. Descriptive analyses were conducted and proportions as well as absolute frequencies are presented for categorical variables. Continuous measures were not normally distributed and median and quartiles are presented for these variables. $\chi^{2}$ tests of independence were used for categorical variables and Mann-Whitney tests for numeric variables. The association between initial MTS category and mortality was assessed using Cox proportional hazards regression. MTS category was included in the regression models as a binary predictor (ie, urgent vs non-urgent). Sensitivity analyses were conducted and are detailed in online supplement 1 to this article.

\section{Patient and public involvement}

Study participants and members of the public were not involved in the development of the study protocol.

\section{Ethical considerations}

This study was conducted in accordance with the Declaration of Helsinki and Good Scientific Practice Guidelines.

\section{RESULTS}

\section{Study population}

A total of 20461 adult non-surgical ED presentations were recorded during the study period and $1152(5.6 \%)$ were enrolled in the study. Of these, $52.1 \%$ were male $(n=600)$ and $47.9 \%$ female $(\mathrm{n}=552)$, median age was 59 years (IQR: $43-71), 31.1 \%(\mathrm{n}=358)$ had a non-urgent MTS triage category and $66.3 \%(\mathrm{n}=764)$ had an urgent triage category. Thirty patients $(2.6 \%)$ did not receive a triage category on presentation to the ED and these presentations were excluded from analyses stratified by triage category. Further data are presented in table 1.

\section{Diagnoses}

Angina pectoris (ICD-code I20) was the most frequent ED diagnosis overall $(5.2 \%, \mathrm{n}=60)$ and for those with an urgent triage category $(6.5 \%, \mathrm{n}=50)$. For those with a non-urgent triage category, the most common ICD-code diagnoses were gastroenteritis/colitis (A09, 7.0\%, n=25), primary hypertension $(\mathrm{I} 10,3.1 \%, \mathrm{n}=11)$, dizziness and giddiness $(\mathrm{R} 42,3.1 \%, \mathrm{n}=11)$, angina pectoris (I20, 2.8\%, $\mathrm{n}=10)$, epilepsy (G40, 2.2\%, $\mathrm{n}=8$ ) and gastroenteritis/duodenitis (K29, 2.2\%, $\mathrm{n}=8)$. The five most frequent ICD diagnoses for ED presentation and hospital admission are illustrated in online supplement 2 table 1.

\section{In-hospital course and short-term outcome data}

The majority of ED patients were discharged home directly from ED $(57.5 \%, \mathrm{n}=662)$ and $42.5 \% \quad(\mathrm{n}=490)$ required hospital admission. Compared with non-urgent ED presentations, those with an urgent triage category were older (median age 60 vs 56 years, $\mathrm{p}=0.001$ ), were more likely to require hospital admission $(47.8 \%$

Table 1 Patients' demographic characteristics, chief complaints and in-hospital course for all patients, stratified by urgency according to initial triage level based on the German version of the Manchester Triage System: urgent refers to patients with a triage category of yellow, orange or red and non-urgent to patients with a triage category of blue or green

\begin{tabular}{|c|c|c|c|c|c|}
\hline Variable & $\begin{array}{l}\text { All } \\
(n=1152)\end{array}$ & $\begin{array}{l}\text { Non-urgent } \\
(n=358)\end{array}$ & $\begin{array}{l}\text { Urgent } \\
(n=764)\end{array}$ & P value* & $\begin{array}{l}\text { Missing triage } \\
(n=30)\end{array}$ \\
\hline Female \% (n) & $47.9(552)$ & $52.0(186)$ & $46.2(353)$ & 0.042 & $43.3(13)$ \\
\hline Age (years), median (IQR) & $59(43-71)$ & $56(35-71)$ & $60(46-72)$ & 0.001 & $63(34-70)$ \\
\hline BMI, median (IQR) & $25.6(22.5-29.1)$ & $25.4(22.1-28.8)$ & $25.7(22.8-29.4)$ & 0.192 & $25.9(35.4-28.4)$ \\
\hline Chief complaint \% (n) & & & & 0.001 & \\
\hline Chest pain & $11.0(127)$ & $5.9(21)$ & $13.6(104)$ & & $6.7(2)$ \\
\hline Abdominal pain & $12.8(147)$ & $12.3(44)$ & $13.2(101)$ & & $6.7(2)$ \\
\hline Dyspnea & $10.4(120)$ & $8.9(32)$ & $10.9(83)$ & & $16.7(5)$ \\
\hline Headache & $4.0(46)$ & $4.2(15)$ & $3.9(30)$ & & $3.3(1)$ \\
\hline None of these & $61.8(712)$ & $68.7(246)$ & $58.4(446)$ & & $66.7(20)$ \\
\hline Admitted \% (n) & $42.5(490)$ & $29.6(106)$ & $47.8(365)$ & $<0.001$ & $63.3(19)$ \\
\hline \multicolumn{6}{|c|}{ In-hospital course for admitted patients } \\
\hline $\begin{array}{l}\text { Inpatient LOS (days), median } \\
\text { (IQR) }\end{array}$ & $5(2-9)$ & $6(2-9)$ & $5(2-9)$ & 0.685 & $5(3-11)$ \\
\hline ICU stay \% (n) & $13.3(65)$ & $9.4(10)$ & $14.2(52)$ & 0.128 & $15.8(3)$ \\
\hline In-hospital mortality \% (n) & $1.5(17)$ & $0.8(3)$ & $1.6(12)$ & $<0.001$ & $6.7(2)$ \\
\hline
\end{tabular}

The triage category was missing in 30 patients (last column).

${ }^{*} P$ values refer to a comparison of variables between non-urgent and urgent cases.

BMI, body mass index; ICU, intensive care unit; LOS, length of stay. 
Table 2 One-year mortality stratified by sex, age, level of care and chief complaint at admission

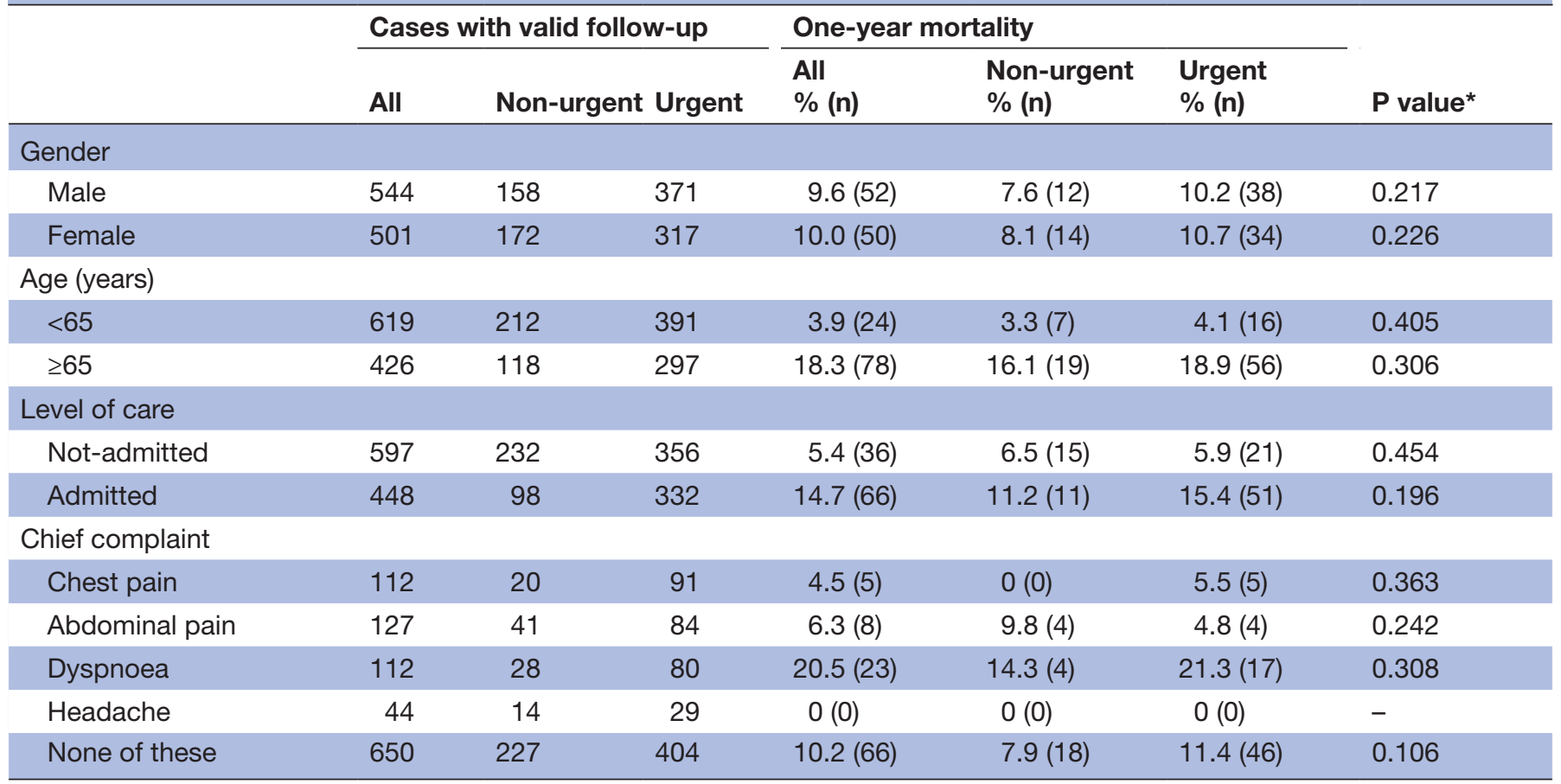

Please note that numbers of non-urgent and urgent cases do not necessarily add up to total numbers as triage category was missing for 30 cases.

${ }^{*} \mathrm{P}$ values refer to a comparison of variables between non-urgent and urgent cases.

vs $29.6 \%)$ and had higher in-hospital mortality $(1.6 \%$ vs $0.8 \%$ ) (table 1). Further details comparing survival status between non-urgent and urgent ED triage categories are provided in online supplement 1 . Specifically, sensitivity analysis of 30-day mortality revealed no significant difference between non-urgent and urgent triage categories $(1.2 \%$ vs $2.2 \%$, respectively, $\mathrm{p}=0.29)$.

\section{One-year outcome data}

One-year mortality status was not available in $9.3 \%$ of all ED presentations $(n=107)$. The total 1 -year mortality was
9.8\% (n=102) (table 2). Compared with non-urgent ED presentations, those with an urgent triage category had similar 1-year mortality ( $10.5 \%$ vs $7.9 \%$, p=0.12) One-year mortality did not show an increasing trend with higher MTS urgency (figure 1). From lowest to highest urgency, mortality rates were blue $(12.5 \%, \mathrm{n}=2)$, green $(7.6 \%$, $\mathrm{n}=24)$, yellow $(9.0 \%, \mathrm{n}=40)$, orange $(13.5 \%, \mathrm{n}=32)$ and red (no deaths).

Cox proportional hazards regression revealed no association between urgency of ED presentation and

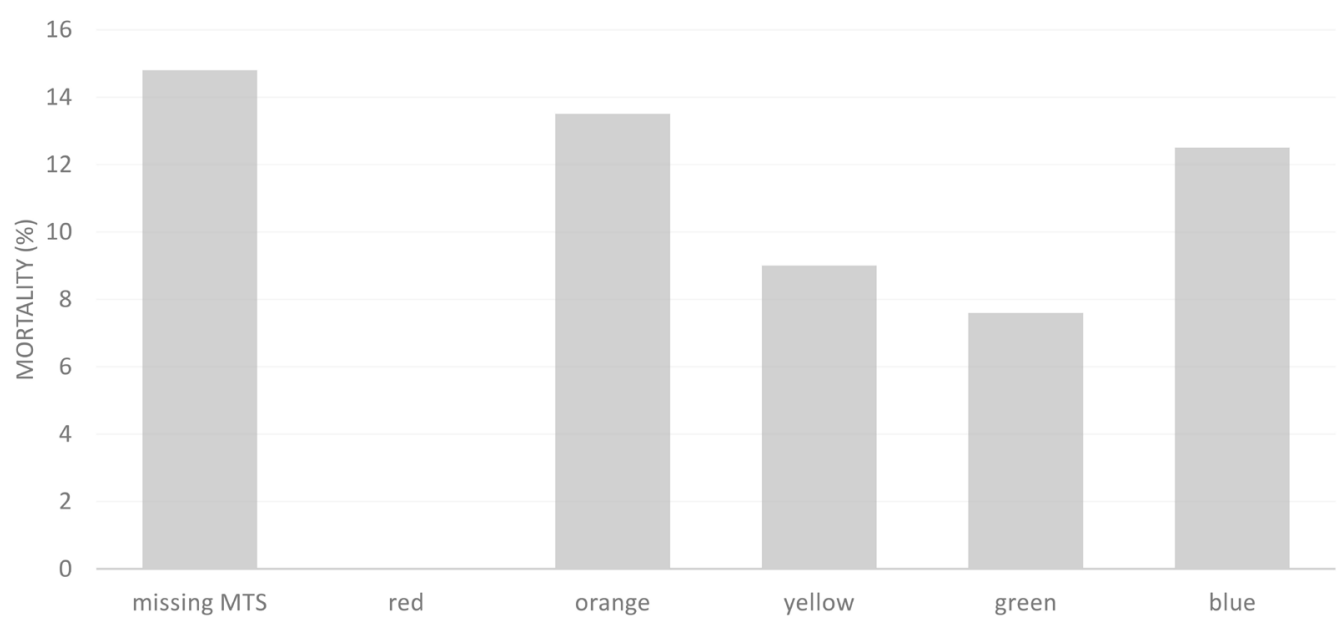

Figure 1 One-year mortality within MTS categories. MTS, Manchester Triage System. 

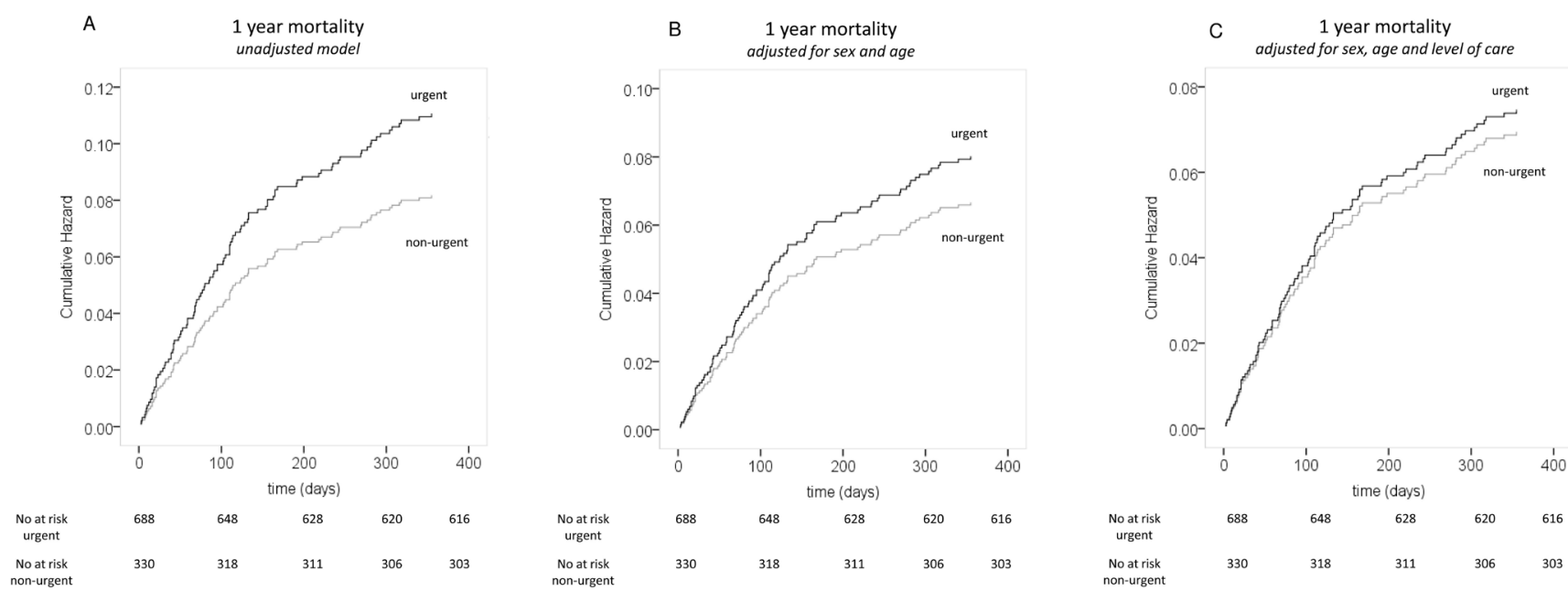

Figure 2 Cumulative hazards of 1-year mortality for urgent and non-urgent cases. (A) Crude cumulative hazards of 1-year mortality for urgent and non-urgent cases. Urgency was defined by the initial triage category (MTS) as urgent (MTS categories red, orange and yellow) or non-urgent (MTS categories green and blue). (B) Cumulative hazards of 1-year mortality when adjusted for sex (male/female), age (<65 years/ $\geq 65$ years). (C) Cumulative hazards of 1-year mortality adjusted for sex (male/ female), age (<65 years/ $\geq 65$ years) and level of care (admitted/not-admitted). MTS, Manchester Triage System.

1-year mortality when analysed as a binary predictor $(\mathrm{HR}=1.35 ; 95 \%$ CI 0.87 to $2.12 ; \mathrm{p}=0.185$ ) (figure 2 and table 3 ) and in both multivariate analyses (model 1$\mathrm{HR}=1.20 ; 95 \%$ CI 0.77 to $1.89 ; \mathrm{p}=0.420$ and model 2 $\mathrm{HR}=1.07 ; 95 \%$ CI 0.679 to $1.699 ; \mathrm{p}=0.759)$. Further details comparing 1-year mortality between non-urgent and urgent ED triage categories are provided in online supplement 1 table 1 . The initial diagnoses for all patients and discharge diagnoses for admitted patients were heterogeneous. Patients with a non-urgent triage category, who died within 1 year, had a variety of diagnoses (online supplement 1 table 2 ).

\section{Sensitivity analyses}

Sensitivity analyses were conducted for mortality at 30 days (online supplement 1 figure 1), potential sampling bias (online supplement 1 table 3 ) and differential loss to follow-up (online supplement 1 part 4).

Table 3 Univariate and multivariate Cox regression models were calculated to evaluate the association between MTScategory at admission and mortality after 1 year

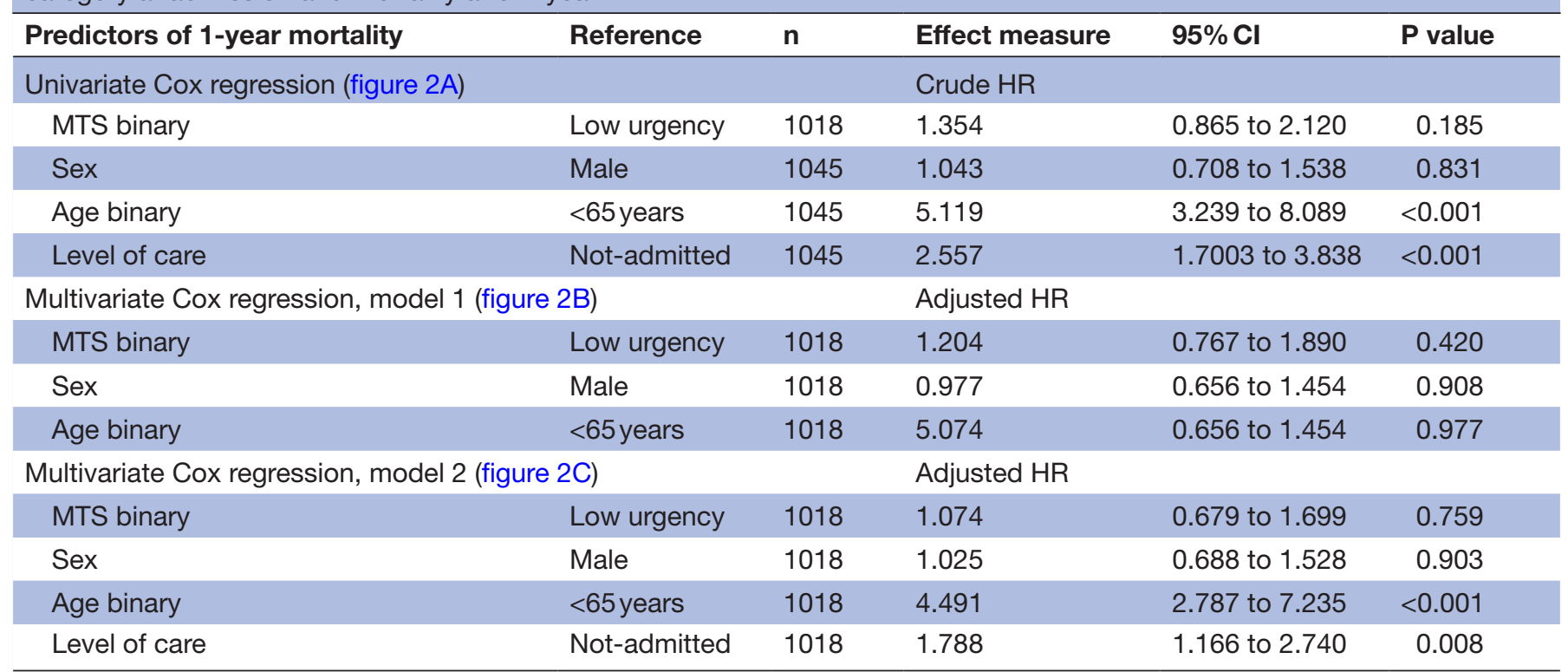

Multivariate HRs were adjusted for age and gender (model 1) and further adjusted for level of care (model 2). The MTS was analysed as binary variable (non-urgent: green and blue MTS-category; urgent: yellow, orange and red MTS-category) in Cox regression analysis. Cases with missing triage were excluded from this analysis.

MTS, Manchester Triage System. 


\section{DISCUSSION}

This is the first study of its kind to examine in-hospital course, diagnoses and mortality during an initial hospital stay, after 30 days and within 1 year among adult, non-surgical ED patients with low urgency according to initial German MTS category in linked routine data from the HIS and the local register of residents. Patients with a non-urgent triage category based on the MTS had severe diagnoses and a high proportion was admitted to hospital and ICU. A considerable number died within 30-days and 1-year mortality was comparable to patients with an urgent triage category. Based on our findings, these patients could not be regarded as GP-type presentations. Redirection of non-urgent cases based on the German MTS to alternative, GP-based care facilities is not recommended as hospital structures and well-trained acute care physicians are needed to assure adequate and rapid treatment of these patients, who are identified as non-urgent based on the German MTS. The German MTS system is thus not suitable as a gatekeeping tool to direct patients to a certain level of care and other tools need to be prospectively validated for this purpose within the German healthcare system.

Some limitations regarding the findings of the current research study should be considered. Primarily, it is uncertain whether some of the outcome measures, such as admittance to hospital and long-term mortality, are appropriate surrogate parameters to determine patients' risk in the acute setting. In Germany, there is no general regulation for admittance to hospital. The decision is taken by the responsible ED physician based on diagnostic findings, comorbidities and patients' status. Alternative study designs have investigated physician's rating of treatment urgency as a primary outcome measure, which allows also for the investigation of overtriage and undertriage. Long-term mortality may not directly indicate an acute need of treatment but still gives an impression of disease severity and is an established outcome parameter to compare patient groups regarding their risk in clinical trials. Furthermore, no long-term outcome data have been investigated in this context so far and the lack of a significant difference in 1-year mortality between non-urgent and urgent patients based on the initial MTS category suggests non-urgent patients do not constitute a low-risk, GP population. The vital status in the register of residents was not available in $9.3 \%$ of all patients $(n=107)$. The vital status of these patients could be non-differentially missing and thus effect measures could be biased by these patients with missing outcome information. We therefore conducted sensitivity analyses to investigate whether there were differences regarding the proportion of patients lost to follow-up in the MTS categories. There was, however, a lower proportion of patients lost to follow-up in the blue MTS category. Thus, mortality could have been higher in this category. Considering the low number of patients with missing outcome information in general, this finding is not likely to have affected the conclusions of our study. Moreover, the participants of our study comprised a selected cohort due to the fact that patients were enrolled based on their ability to give written informed consent. More severe cases were likely to be excluded given the study procedures and thus selection bias may have occurred. To address this concern, data of three consecutive cohorts of 1000 patients each who attended the ED during the study period were extracted from the HIS (online supplement 3). These data show that there appeared to be under sampling of patients in the red MTS category $(0.6 \%-1.0 \%)$. This may explain why no fatal cases occurred in this triage category, which may have affected our findings regarding the comparisons of urgent and non-urgent triage categories. This bias, however, is unlikely to have affected our main findings, which considered non-urgent MTS cases only. Finally, case numbers were too small to identify possible differences regarding all five triage categories and to conduct adjusted analyses for short-term mortality.

Currently, different triage systems are implemented worldwide: the Australasian Triage Scale, ${ }^{7}$ Canadian Triage and Acuity Scale, ${ }^{8}$ Emergency Severity Index $\left(\mathrm{ESI}^{9}\right)$ and the MTS. ${ }^{10}$ While the MTS was originally developed in the UK, it is now widely implemented in EDs across Europe and North America and a German translation has been available since 2008. ${ }^{11} 12$ In Germany, the German version of the MTS and ESI are predominately utilised. The MTS has a good reliability ( $\kappa$-statistics $0.31-0.62)^{12}$ and several studies investigated the association between the MTS and short-term outcomes; however, only a few studies investigated the association between the MTS and severe diagnoses, mortality and long-term mortality.

When comparing the proportion of patients admitted to hospital, other studies found lower admission rates of $13 \%-16 \%$ in patients with blue and green MTS categories. ${ }^{61113}$ One explanation for these lower admission rates may be that these studies investigated non-surgical as well as surgical ED patients. Surgical ED patients in general show a lower admission rate. Regarding ICU admission and LOS, Steiner et al found an association between admission to ICU and MTS category but no differences in length of hospital stay regarding different MTS categories. ${ }^{11}$ Gräff et al reported that $0.17 \%$ of patients with blue triage category and $0.28 \%$ of patients with green triage category were admitted to ICU. ${ }^{6}$ Again, the difference between these results and ICU admission in the current study (2.8\% of all patients with lower urgency) may be explained by our exclusion of surgical patients. Hsia and Niedzwiecki investigated non-urgent ED visits based on the triage score utilised in the National Hospital Ambulatory Medical Care Survey. ${ }^{14}$ Based on their results, $1.3 \%$ were admitted to critical care units and the authors also reported a high utilisation of diagnostic tests or procedures. There is little evidence into underlying diagnoses for patients with low urgency according to the MTS. Other studies confirm a considerable proportion of cardiovascular and neurological diagnoses in non-urgent patients and broad overlap in the top 10 diagnoses between non-urgent and urgent patients. ${ }^{11}$ Length of 
hospital stay did not differ significantly between urgent and non-urgent patients in most studies. ${ }^{14}$

When considering short-term mortality, other studies confirm a non-trivial number of fatal events during the initial hospital stay $(2 \%)$ and 30 days $(4.8 \%) .{ }^{6}{ }^{11}$ Conflicting to our results, other studies found that the MTS was a significant predictor of 30-day mortality in a German patient cohort. ${ }^{6}$ A sensitivity analysis of 30-day mortality in our study revealed no significant association (online supplement 1 figure 1). Some studies investigated ED mortality and found no death ${ }^{13}$ and 1 out of 321539 patients, respectively. ${ }^{15}$ In summary, other studies show that patients with a non-urgent triage category may still constitute a population at risk. These patients require a thorough diagnostic workup, may have severe diagnoses and include a relevant proportion in need of admission to hospital or ICUs with a non-negligible risk of mortality.

\section{Clinical significance}

Independent of triage purpose, the performance of one single initial triage does not address the fact that a patient's status must be considered to be a dynamic state and, especially in the emergency setting, could worsen. ${ }^{16}$ This may be a clinical explanation for fatal events in non-urgent cases. Another explanation may be a low sensitivity of the initial triage to predict patient outcome. Parenti et al summarised 12 studies on validity and reliability of the MTS. ${ }^{17}$ The authors concluded that the safety of the MTS is limited, even for the ED setting, due to a high rate of under triage $(11 \%-25 \%)$ and a low sensitivity $(16 \%-$ $63 \%)$ and suggested that the MTS needs to be adapted to improve patient safety even for the original purpose of determining the time until first physician contact. These findings underline that the MTS is not eligible for gatekeeping purposes and may expose patients to avoidable danger. We thus strongly recommend that also patients with low urgency according to the MTS must still remain under close surveillance in a hospital-based setting during this waiting period to ensure the recognition and adequate handling of a worsening physical patient status in a setting with well-trained nurses and physicians, and necessary equipment and resources, so any deterioration can be addressed rapidly and adequately. A retriage is highly recommended, even in the ED setting, if initially determined waiting times could not be met.

\section{Alternative algorithms for patient redirection}

Other triage tools, however, showed a higher validity and reliability as compared with the MTS. One of these tools, the ESI, is also widely implemented in Germany. The ESI also showed a better correlation with short-term risk and resource utilisation as compared with the MTS and may be more eligible as a basis to develop such a gatekeeping tool for the identification of patients who could be safely redirected to GP care. ${ }^{12}$ Also, the implementation of other tools like the SMASS, which is used for patient navigation purposes in Switzerland, could be discussed. However, it needs to be highlighted that none of these tools should be implemented without a prospective validation within the German healthcare system. ${ }^{18}$

There is currently no tool available which has been validated for the German healthcare system. Further prospective research is necessary to assure patients' safety when such systems should be implemented in Germany. Some available tools could potentially be adapted for, or implemented in, the German healthcare system. ${ }^{19-23}$ For Germany, however, little evidence is available on the number, characteristics, diagnoses and outcome of ED patients in general. ${ }^{1}$ Thus, no conclusions can be made regarding the comparability of the German ED population with patients seeking ED care in other countries.

\section{CONCLUSIONS}

Patients with a non-urgent triage category based on the German version of the MTS had severe diagnoses and a high proportion was admitted to hospital and ICU. Shortterm as well as long-term mortality was not significantly different when compared with patients with an urgent triage category. Based on our findings, a redirection of non-urgent cases, as identified by the German version of the MTS, to alternative, GP-based care facilities is not recommended as hospital structures and well-trained acute care physicians are needed to ensure adequate and rapid treatment of these patients. The German MTS is thus not suitable as a gatekeeping tool to direct patients to a certain level of care. Nevertheless, gatekeeping measures may be effective to reduce patient numbers in overcrowded EDs. Alternative algorithms need to be developed and prospectively validated for this purpose within the German healthcare system, to facilitate safe and reasonable patient navigation.

\section{Author affiliations}

${ }^{1}$ Australian Institute of Tropical Health and Medicine, College of Public Health Medical and Veterinary Sciences, Centre for Chronic Disease Prevention, James Cook University, Cairns, Queensland, Australia

${ }^{2}$ Emergency and Acute Medicine (CVK, CCM), Charite Universitätsmedizin Berlin, Berlin, Germany

${ }^{3}$ Department of Trauma Surgery, Otto-von-Guericke University Magdeburg, Magdeburg, Germany

${ }^{4}$ Institute of Medical Sociology and Rehabilitation Science, Charité Universitätsmedizin Berlin, Berlin, Germany

Contributors AS was involved in the conception and design of the study, the acquisition, analysis and interpretation of data, drafted the manuscript, approved the final version to be published, is accountable for all aspects of the work and serves as guarantor for the manuscript. FG, LH, FT, JF, MB and TL were involved in the interpretation of data, critically revised the manuscript for important intellectual content, approved the final version to be published and agreed to be accountable for all aspects of the work. JS was involved in the conception and design of the study, the acquisition and interpretation of data, critically revised the manuscript for important intellectual content, approved the final version to be published and agreed to be accountable for all aspects of the work. MM was involved in the conception, analysis and design of the study, the acquisition and interpretation of data, critically revised the manuscript for important intellectual content, approved the final version to be published, agreed to be accountable for all aspects of the work and serves as guarantor. The corresponding author attests that all listed authors meet authorship criteria and that no others meeting the criteria have been omitted. 
Funding This study was supported by Thermofisher Scientific. The sponsor was not involved in study design, collection, analysis, interpretation of data, the content of this report and the decision to submit the paper for publication.

Competing interests This study was supported by Thermofisher Scientific. AS, JS, JF, MB, TL and MM report grants from Thermoficher Scientific, during the conduct of the study; grants from Roche Diagnostics, grants from Thermofisher Scientific, grants from Novartis, grants from Cardiorentis, grants from Governmenta institutions, from Foundations, outside the submitted work. MM reports grants from Thermoficher Scientific, during the conduct of the study; grants and personal fees from Roche Diagnostics, grants and personal fees from Thermofisher Scientific, grants and personal fees from Novartis, grants from Cardiorentis, grants from Governmental institutions, grants from Foundations, personal fees from Bayer Healthcare, personal fees from Boehringer Ingelheim, personal fees from Daiichi Sankyo, personal fees from Astra Yeneca, outside the submitted work. FG, LH and FT have nothing to disclose.

Patient consent for publication Not required.

Ethics approval The study protocol was ethically approved by the institutional review board of the Charité Universitätsmedizin Berlin (EA2/121/10).

Provenance and peer review Not commissioned; externally peer reviewed. Data sharing statement Relevant data could be shared on reasonable request. Open access This is an open access article distributed in accordance with the Creative Commons Attribution Non Commercial (CC BY-NC 4.0) license, which permits others to distribute, remix, adapt, build upon this work non-commercially, and license their derivative works on different terms, provided the original work is properly cited, appropriate credit is given, any changes made indicated, and the use is non-commercial. See: http://creativecommons.org/licenses/by-nc/4.0/.

\section{REFERENCES}

1. Berchet C. Emergency Care Services: Trends, Drivers and Interventions to Manage the Demand. OECD Health Working Papers. 2015:83.

2. Van den Heede K, Van de Voorde C. Interventions to reduce emergency department utilisation: a review of reviews. Health Policy 2016;120:1337-49.

3. Koalitionsvertrag zwischen, C, CSU und SPD. Ein neuer Aufbruch für Europa Eine neue Dynamik für Deutschland Ein neuer Zusammenhalt für unser Land. 2018.

4. Sachverständigenrat Z. Die Zukunft der Notfallversorgung in Deutschland. 2017.

5. Tobias Herrmann CK, Seyderhelm A, Broge B, et al. Instrumente und Methoden zur Ersteinschätzung von Notfallpatienten. Bestandaufnahme und Konzeptentwicklung für die kassenärztliche Notfallversorgung. Göttingen. aQua-Institut für angewandte
Qualitätsförderung und Forschung im Gesundheitswesen $\mathrm{GmbH}$. 2017.

6. Gräff I, Goldschmidt B, Glien P, et al. The German Version of the Manchester Triage System and its quality criteria--first assessment of validity and reliability. PLoS One 2014;9:e88995.

7. Australasian College for Emergency, M. The Australasian Triage Scale. Emerg Med (Fremantle). 2002;14:335-6.

8. Bullard MJ, Musgrave E, Warren D, et al. Revisions to the Canadian Emergency Department Triage and Acuity Scale (CTAS) Guidelines 2016. CJEM 2017;19(S2):S18-27.

9. Shelton R. The Emergency Severity Index 5-level triage system. Dimens Crit Care Nurs 2009;28:9-12.

10. Schellein O, Ludwig-Pistor F, Bremerich DH, et al. Process optimization in the interdisciplinary emergency department]. Anaesthesist 2009;58:163-70.

11. Steiner D, Renetseder F, Kutz A, et al. Performance of the Manchester Triage System in Adult Medical Emergency Patients: A Prospective Cohort Study. J Emerg Med 2016;50:678-89.

12. Christ M, Grossmann F, Winter D, et al. Modern triage in the emergency department. Dtsch Arztebl Int 2010;107:892-8.

13. van der Wulp I, Schrijvers AJ, van Stel HF. Predicting admission and mortality with the Emergency Severity Index and the Manchester Triage System: a retrospective observational study. Emerg Med J 2009;26:506-9.

14. Hsia RYF, Niedzwiecki A. M Urgent care needs among nonurgent visits to the Emergency Department. JAMA 2016;176:852-4.

15. Martins HM, Cuña LM, Freitas P. Is Manchester (MTS) more than a triage system? A study of its association with mortality and admission to a large Portuguese hospital. Emerg Med $J$ 2009;26:183-6.

16. Robertson-Steel I. Evolution of triage systems. Emerg Med J 2006;23:154-5.

17. Parenti N, Reggiani ML, lannone $P$, et al. A systematic review on the validity and reliability of an emergency department triage scale, the Manchester Triage System. Int J Nurs Stud 2014;51:1062-9.

18. Pin MD, Somasundaran C;, Gräff R; , et al. Positionspaper zur Ersteinschätzung in integrierten Notfallzentren. Notfall Rettungsmed 2018.

19. Bunn F, Byrne G, Kendall S. The effects of telephone consultation and triage on healthcare use and patient satisfaction: a systematic review. Br J Gen Pract 2005;55:956-61.

20. Group MT. Emergency triage: telephone triage and advice: Wiley, 2015.

21. Huibers L, Moth G, Carlsen AH, et al. Telephone triage by GPs in outof-hours primary care in Denmark: a prospective observational study of efficiency and relevance. Br J Gen Pract 2016;66:e667-73.

22. Kantonen J, Menezes R, Heinänen T, et al. Impact of the ABCDE triage in primary care emergency department on the number of patient visits to different parts of the health care system in Espoo City. BMC Emerg Med 2012;12:2.

23. Schaufelberger M FP, Derkx H, Meer A. Red flags - Expertenkonsens - Alarmsymptome der Mediyin. Editions D\&F GmbH, Suisse. 2013:1. 